\title{
RGMa regulates CCL5 expression via the BMP receptor in experimental autoimmune encephalomyelitis mice and endothelial cells
}

\author{
SHI TANG ${ }^{1 *}$, BAO SU $^{2 *}$, TAO TAO $^{3}$, WEIPING YAN ${ }^{4}$, RONGRONG ZHANG ${ }^{1}$, \\ XINYUE QIN $^{1}$ and JINZHOU FENG ${ }^{1}$
}

\begin{abstract}
Departments of ${ }^{1}$ Neurology and ${ }^{2}$ Orthopedics, The First Affiliated Hospital of Chongqing Medical University, Chongqing 400016; ${ }^{3}$ Department of Neurology, The Affiliated Hospital of Southwest Medical University, Luzhou, Sichuan 646000; ${ }^{4}$ Department of Neurology, Guangrao District People's Hospital, Dongying, Shandong 257300, P.R. China
\end{abstract}

Received May 18, 2021; Accepted December 17, 2021

DOI: $10.3892 / \mathrm{mmr} .2022 .12601$

\begin{abstract}
Multiple sclerosis (MS) is a demyelinating disease of the central nervous system (CNS). Repulsive guidance molecule a (RGMa) has been indicated to act as a bone morphogenetic protein (BMP) co-receptor, enhancing BMP signalling activity. However, the role and downstream pathways of the BMP signalling pathway mediated by RGMa have yet to be fully elucidated. A recent study revealed that $\mathrm{C}-\mathrm{C}$ motif chemokine ligand 5 (CCL5) has a major role in the pathogenesis of MS via the recruitment of macrophages and T-lymphocytes into the CNS. The present study aimed to evaluate whether RGMa regulates CCL5 via the BMP pathway in MS. The results demonstrated that RGMa regulated CCL5 expression in a BMP ligand-dependent manner in experimental autoimmune encephalomyelitis (EAE) mice in vivo and in endothelial cells in vitro. First, specific inhibition of the expression of RGMa via RNA interference led to a significant reduction of the expression of RGMa and this was associated with a significant delay of EAE, an alleviated disease course and downregulation of CCL5 expression at both the protein and mRNA levels. Furthermore, exogenous noggin, an extracellular antagonist of BMP ligand, abolished the induction effect of RGMa on CCL5 in endothelial cells. Taken together, these results suggested that RGMa is an important regulator
\end{abstract}

Correspondence to: Professor Xinyue Qin or Dr Jinzhou Feng, Department of Neurology, The First Affiliated Hospital of Chongqing Medical University, 1 Youyi Road, Chongqing 400016, P.R. China

E-mail: qinxinyuecqchina@hotmail.com

E-mail: fengjinzhou@hotmail.com

*Contributed equally

Key words: repulsive guidance molecule a, $\mathrm{C}-\mathrm{C}$ motif chemokine ligand 5 , bone morphogenetic protein receptor, experimental autoimmune encephalomyelitis, multiple sclerosis of MS and inflammatory mediators such as CCL5, and the present results should prove to be useful in terms of further elucidating the RGMa-BMP receptor signalling pathway and the pathogenesis of RGMa on MS as far as the involvement of blood-brain barrier permeability is concerned.

\section{Introduction}

Multiple sclerosis (MS) is the most common inflammatory demyelinating disease of the central nervous system (CNS), which most commonly affects young adults. Repulsive guidance molecule a (RGMa) is a glycosylphosphatidylinositolanchored membrane protein that fulfils an important role in axonal outgrowth $(1,2)$. Recent evidence has suggested that RGMa has a critical role in the pathogenesis of MS (3). Specifically, inhibition of RGMa was indicated to attenuate clinical symptoms and reduce the ability of inflammatory cells to invade the CNS (3), and also to modulate T-cell activation in myelin oligodendrocyte glycoprotein (MOG)-induced experimental autoimmune encephalomyelitis (EAE) mice (4), findings which demonstrated that targeting RGMa may be a novel therapeutic strategy in MS. RGMa expressed in endothelial cells suppressed endothelial tube formation, angiogenesis and neovascularization, leading to alterations in the permeability of the blood-brain barrier (BBB) in the pathogenesis of MS (5), a phenomenon that is among the earliest observed cerebrovascular abnormalities to occur in MS (6). A previous study published by our group also indicated that RGMa was able to suppress angiogenesis (7). Therefore, the aim of the present study was to further explore the downstream signalling pathway featuring RGMa in endothelial cells and in MS.

A previous study suggested that chemokines and chemokine receptors participate in the recruitment of macrophages and $\mathrm{T}$ lymphocytes into the CNS and this has been considered as the most important mechanism involved in the pathogenesis of MS (8). Chemokine ligand 5 (CCL5) serves a major role in several inflammatory diseases due to its ability to control the migration of memory B-lymphocytes, monocytes, macrophages and eosinophils into the CNS $(9,10)$. The level of CCL5 was indicated to increase both in EAE mice and in patients with 
MS, and this molecule has been demonstrated to be involved in the pathophysiology of MS (9). Knocking out the CCL5 receptor (CCR5) was indicated to lead to marked improvements in the clinical scoring and neurological functions in EAE mice, and also suppressed the expression of inflammatory mediators, including IL- $1 \beta, \mathrm{TNF}-\alpha$, IFN- $\gamma$ and monocyte chemoattractant protein-1 (MCP-1) (11). However, how CCL5 participates in the underlying mechanism of the pathogenesis of MS and how it is regulated has yet to be fully elucidated. The present study explored whether RGMa regulates CCL5 expression in a BMP ligand-dependent manner.

\section{Materials and methods}

Experimental animals. Female C57BL/6 mice (weight, 18-20 g; age, 8-10 weeks; $n=112$ ) were obtained from the Experimental Animal Centre of Chongqing Medical University. All mice were housed in groups (five mice per cage) in a colony room at $22 \pm 2^{\circ} \mathrm{C}$ and $45 \pm 10 \%$ humidity under a reverse 12 -h light/dark cycle and had ad libitum access to food and water. All animal procedures were approved by the Ethics Committee of the First Affiliated Hospital of Chongqing Medical University (Chongqing, China) and all procedures were performed in accordance with the Guide for the Care and Use of Laboratory Animals (12).

Induction of EAE and clinical scoring. EAE was induced in female C57 BL/6 mice by subcutaneously injecting $100 \mu \mathrm{g}$ MOG 35-55 peptide (Sigma-Aldrich; Merck KGaA) emulsified in Complete ${ }^{\mathrm{TM}}$ Freund's adjuvant including Mycobacterium tuberculosis (Sigma-Aldrich; Merck KGaA) into the scapular region following anaesthesia with $2 \%$ isoflurane. In addition, intraperitoneal injections of $200 \mathrm{ng}$ of pertussis toxin (Sigma-Aldrich; Merck KGaA) were performed on days 0 and 2 . The state of EAE in the mice was monitored daily to assess the clinical scores of disease leading up to the completion of the experiment using the following scoring system: 1, Complete tail atony; 2, hindlimb weakness; 3 , hindlimb paralysis; 4, complete hind limb paralysis and front limb weakness; and 5, moribund (13). If mice with EAE were deemed to be within two scores, a 0.5 value was added to the lower of the clinical scores. The bodyweight of the mice was also monitored daily.

Animal treatment. RGMa-specific recombinant adenovirus rAd5-short hairpin (sh)RNA-RGMa and the same empty carrier recombinant adenovirus rAd5-HK were supplied by Wuhan Genesil Biotechnology Co., Ltd. The virus was amplified in 293 cells and purified using a Sartorius Vivapure ${ }^{\circledR}$ Adeno PACK ${ }^{\mathrm{TM}} 20$ (Sartorius AG). Details of the titres, delivery efficacy and toxicity of recombinant adenovirus were the same as those described in a previously published study by our group (14). Mice were randomly divided into the EAE control group, the rAd5-HK control group and the rAd5-shRNA-RGMa group [RGMa RNA interference (RNAi) group] ( $\mathrm{n}=10$ mice in each group). The rAd5-HK and rAd5-shRNA-RGMa groups were individually treated by intracerebroventricular injection on a stereotaxic instrument at 12 days post-immunization after anaesthesia (p.i.) $(15,16)$. The injection rate was $0.3 \mu \mathrm{l} / \mathrm{min}$ and the total volume was $2 \mu \mathrm{l}$ for each site. At the end of the injection, the microinjector was kept immobile for 5 min prior to withdrawal. $2 \%$ isoflurane was used for induction and maintenance of anesthesia. Any of the following criteria were endpoints for immediate premature euthanasia according to a previous consensus on EAE model: i) Paralyzed in all four limbs and not mentally alert; ii) alert but exhibited paralysis in all four limbs for $>24 \mathrm{~h}$; and iii) Dermatitis or posthitis (penile inflammation) with ulceration from excessive urinary moisture. No EAE animals met endpoint criteria for disease severity prior to the end of the experiment in this study. Mice were sacrificed using cervical dislocation under anesthesia at the designated timepoint on the examination day (mice used for the immunofluorescence experiment were subjected to cardiac perfusion after anesthesia). Absence of a corneal reflex, failure to detect respiration and absence of a heartbeat for a period of $>10 \mathrm{~min}$ were used to confirm death.

Cell culture. bEnd.3 cells [American Type Culture Collection (ATCC) ${ }^{\circledR}$ CRL2299 ${ }^{\text {TM }}$; ATCC] were chosen for use in the present study and grown in ATCC-formulated Dulbecco's modified Eagle's medium (Gibco; Thermo Fisher Scientific, Inc.) containing $10 \%$ foetal bovine serum (Atlanta Biologicals, Inc.) in a humidified incubator at $37^{\circ} \mathrm{C}$ in an atmosphere with $5 \% \mathrm{CO}_{2}$. The medium was changed every $48 \mathrm{~h}$. Cells were plated on culture dishes at a density of $4 \times 10^{4}$ cells $/ \mathrm{ml}$. At $48 \mathrm{~h}$ after seeding, bEnd.3 cells were treated with RGMa (R\&D Systems, Inc.; $2 \mu \mathrm{g} / \mathrm{ml}$ ) for $0-4 \mathrm{~h}$ and subsequently incubated with noggin (R\&D Systems, Inc.; $500 \mathrm{ng} / \mathrm{ml}$ ) overnight.

ELISA. CCL5 (cat. no. SEA116Po; Wuhan USCN Business Co., Ltd.) was used for measuring the concentration of chemokine ligand in the serum of mice. Samples were measured in duplicate using ELISA kits following the manufacturer's recommendations. The results are expressed as the cytokine concentration in $\mathrm{pg} / \mathrm{ml}$.

Western blot analysis. Briefly, tissues were lysed in RIPA (cat. no. P0013B; Beyotime Institute of Biotechnology) with protease and phosphatase inhibitor cocktail (cat. no. 78441; Thermo Fisher Scientific, Inc.) and cleared of debris by centrifugation at $14,000 \times \mathrm{g}$ for $15 \mathrm{~min}$ at $4^{\circ} \mathrm{C}$. The protein concentration was determined with a BCA Protein Assay kit (cat. no. P0011; Beyotime Institute of Biotechnology). Equal amounts of sample protein $(20 \mu \mathrm{g})$ were separated via $10 \%$ SDS-PAGE and transferred to PVDF membranes (MilliporeSigma). The membranes were probed using antibodies that specifically recognized RGMa (cat. no. sc-46484; 1:1,000 dilution; Santa Cruz Biotechnology, Inc.) or $\beta$-actin (cat. no. sc-58673; 1:2,000; Santa Cruz Biotechnology, Inc.) at $4^{\circ} \mathrm{C}$ overnight. After three washes in PBS containing $0.1 \%$ Tween-20, blots were probed with the HRP-conjugated donkey anti-goat IgG (1:1,000; cat. no. A0181; Beyotime Institute of Biotechnology) or goat anti-mouse IgG (cat. no. A0216; 1:1,000; Beyotime Institute of Biotechnology) secondary antibodies for $2 \mathrm{~h}$ at room temperature. Protein bands were visualized using an ECL (cat. no. 32109, Thermo Fisher Scientific, Inc.) plus Western blotting detection system (Biorad ChemiDoc MP). $\beta$-actin was used as a loading control. Relative protein expression levels were reflected by the band density of target proteins relative to $\beta$-actin. 
Immunofluorescence. Mice were anesthetized with $2 \%$ isoflurane and perfused with PBS and $4 \%$ buffered paraformaldehyde (PFA) for sacrifice. The brains were then post-fixed in $4 \%$ PFA for $24 \mathrm{~h}$ and subsequently embedded in paraffin and coronally cut into $4-\mu \mathrm{m}$ sections for analysis. Paraffin-embedded sections were deparaffinized, rehydrated in a graded series of ethanol and then incubated in $\mathrm{H}_{2} \mathrm{O}_{2}(0.3 \%$ solution) for $15 \mathrm{~min}$ at room temperature. For antigen retrieval, sections were treated with $10 \mathrm{mmol} / \mathrm{l}$ sodium citrate buffer ( $\mathrm{pH} \mathrm{6.0)}$ and heated in a microwave oven for $20 \mathrm{~min}$. Tissues were permeabilized with $0.5 \%$ Triton $\mathrm{X}-100$, and the sections were then incubated in 5\% goat serum (cat. no. C0265; Beyotime Institute of Biotechnology) for $30 \mathrm{~min}$ at room temperature. Subsequently, the sections were incubated with CCL5 primary antibodies (cat. no. AF478; 1:200 dilution; R\&D Systems, Inc.) at $4{ }^{\circ} \mathrm{C}$ overnight. Sections were then washed and incubated with tetramethylrhodamine isothiocyanate-conjugated IgG (cat. no. ab7686; 1:100 dilution; Abcam) in the dark for $1 \mathrm{~h}$ at $37^{\circ} \mathrm{C}$, prior to the addition of DAPI for $5 \mathrm{~min}$. The resulting sections were observed under a fluorescence microscope (TCS SP5; Leica Microsystems $\mathrm{GmbH}$ ). Images were processed with ImagePro 6.0 software (Media Cybernetics, Inc.).

Total RNA extraction and reverse transcription-quantitative $(R T-q) P C R$. Total RNA was purified from mouse whole spinal cord or b end. 3 cells using TRIzol ${ }^{\circledR}$ reagent (Takara Biotechnology Co., Ltd.) according to the manufacturer's protocol. The total RNA sample was stored at $-80^{\circ} \mathrm{C}$ until required. The RNA concentration was determined by measuring the absorption at $260 \mathrm{~nm}$ on a spectrophotometer and the integrity of the RNA was assessed by running mini-agarose gel electrophoresis. RT was performed using an RT kit (cat. no. RR047A; Takara Biotechnology Co., Ltd.) according to the manufacturer's protocol. Oligo-dT primers were used for generating first-strand cDNA in a reaction mix of $20 \mu \mathrm{l}$. Reactions were performed using SYBR ${ }^{\circledR}$ Green PCR Master Mix (cat. no. RR820A; Takara Biotechnology Co., Ltd.) in a real-time PCR apparatus (iCycler iQ5; Bio-Rad Laboratories, Inc.). The sequences of the primers were as follows: mouse-CCL5 gene sense primer, 5'-AGCCCTCGC TGTCATCCT-3' and antisense primer, 5'-CACTTGGCG GTTCTTTCG-3'; for the internal control, GAPDH sense primer, 5'-CCTACCCCCAATGTATCCGTTGTG-3' and antisense primer, 5'-GGAGGAATGGGAGTTGCTGTTGAA-3' (Sangon Biotech Co., Ltd.). The thermocycling conditions for all reactions commenced with a denaturation step at $95^{\circ} \mathrm{C}$ for $10 \mathrm{~min}$, followed by 40 cycles at $95^{\circ} \mathrm{C}$ for $15 \mathrm{sec}$ and $60^{\circ} \mathrm{C}$ for $60 \mathrm{sec}$. Each sample from one set of cells was analyzed by qPCR in quadruplicate and three sets of cells were used for the RT-qPCR assays. Results were quantified using the comparative quantification cycle $(\mathrm{Cq})$ method, $2^{-\Delta \Delta \mathrm{Cq}}(17)$. All calculated concentrations of the target gene were divided by the endogenous reference (GAPDH) to obtain the normalized CCL5 expression values (18).

Histopathological analysis. For the histological evaluation of the samples, PFA-fixed, paraffin-embedded sections of the spinal cord were stained with hematoxylin and eosin to assess the level of inflammation. For each mouse, 20-30 transverse section samples were examined from the cervical to thoracic spinal cord. Slices were evaluated by an experienced pathologist in a blinded manner and the extent of inflammation (specifically, the inflammatory index) was determined as follows: 0, no inflammation; 1, cellular infiltration only in the perivascular areas and meninges; 2 , mild cellular infiltration in the parenchyma; 3 , moderate cellular infiltration in the parenchyma; 4 , severe cellular infiltration in the parenchyma (3).

Statistical analysis. The means of two groups of samples were compared using the unpaired t-test. Other statistical comparisons between groups were performed by using one-way multiple-range ANOVA and Tukey's post-hoc test for multiple comparisons. All results were analyzed using GraphPad Prism version 8.0 (GraphPad Software, Inc.). $\mathrm{P}<0.05$ was considered to indicate a statistically significant difference.

\section{Results}

RGMa RNAi significantly inhibits the expression of RGM and is associated with a significant delay of EAE and a markedly alleviated disease course. As detailed in the Materials and methods section, the experimental mice were divided into three groups: The EAE group, the RGMa RNAi group and the rAd5-HK control group. Immunized mice that followed a monophasic course were characterized by ataxia and hind-limb paralysis associated with weight loss and faecal and urinary incontinence. The expression of RGMa was indicated to be significantly lower in the RGMa RNAi group compared with that in the EAE and rAd5-HK control groups on day 21 p.i. $(\mathrm{P}<0.01$; Fig. 1). No significant differences in the incidence of EAE in mice were observed when comparing between the EAE group $(75 \%, 9 / 12)$ and the rAd5-HK control group $(83.3 \%, 10 / 12)\left(\chi^{2}=0.253, \mathrm{P}=0.615\right)$. RGMa RNAi treatment, however, only led to a slight reduction in the incidence rate $(58.3 \%, 7 / 12)$, while it was not significantly different from that in the rAd5-HK control group $\left(\chi^{2}=1.815, \mathrm{P}=0.178\right.$; Table I). RGMa RNAi mice exhibited a delayed onset of EAE at day 11 p.i. compared with both the EAE group (day 8) and the rAd5-HK control group (day 9). Furthermore, RGMa RNAi treatment significantly reduced the daily mean neurological score for EAE from day 11 p.i. until day 21 p.i. (Fig. 2A), whereas the maximal mean score was significantly decreased in the RGMa RNAi group compared with that in the EAE group ( $3.4 \pm 0.5$ vs. $2.3 \pm 0.3, \mathrm{P}=0.034$; Table I). This result was related to the finding that RGMa RNAi treatment led to a marked reduction in the infiltration of cells in the spinal cord at 21 days p.i. (Fig. 2B and C). These results indicated that RGMa RNAi ameliorated the clinical severity of EAE in the model mice and also led to improvements in the neurological functional in EAE mice.

CCL5 expression is upregulated as the development of EAE takes its course. To evaluate the expression of CCL5 at different stages during the disease progression of EAE mice, the CCL5 mRNA and protein levels in the spinal cord of EAE mice were measured by RT-qPCR and ELISA, respectively, at $0,7,14$ and 21 days p.i. The results obtained revealed a significant positive association between the expression levels of CCL5 mRNA and CCL5 protein over the total duration of 
Table I. Incidence and severity of EAE in mice in each group.

\begin{tabular}{lcccc}
\hline Group & Incidence & P-value & Maximal mean neurological score & P-value \\
\hline EAE group & $9 / 12(75)$ & NA & $3.4 \pm 0.5$ & NA \\
rAd5-HK control & $10 / 12(83.3)$ & 0.615 & $3.2 \pm 0.6$ & 0.926 \\
RGMa RNAi & $7 / 12(58.3)$ & 0.178 & $2.3 \pm 0.3$ & 0.034 \\
\hline
\end{tabular}

Values are expressed as n/total $(\%)$ or the mean \pm standard deviation. NA, not applicable; RGMa, repulsive guidance molecule a; EAE, experimental autoimmune encephalomyelitis; RNAi, RNA interference.

A

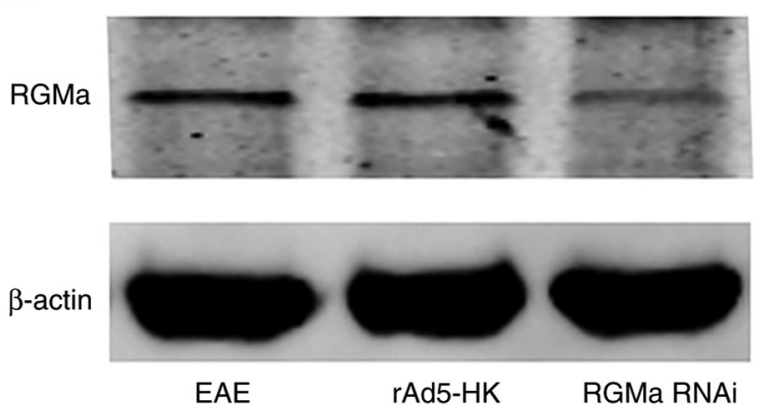

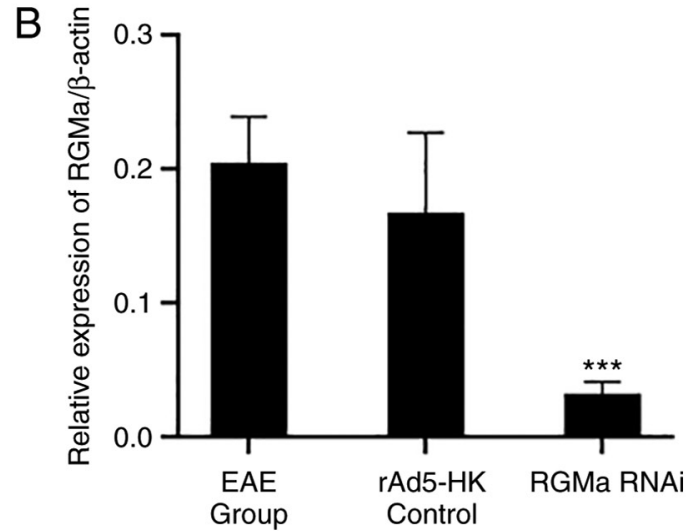

Figure 1. Western blot analysis of RGMa expression in the spinal cord of EAE mice 21 days p.i. RGMa RNAi significantly inhibited the expression of RGMa in the RGMa RNAi group compared with the EAE group $(\mathrm{P}<0.001)$. No significant differences, however, were identified between the EAE and rAd5-HK control groups $(\mathrm{P}=0.134)$. Quantitative analysis of RGMa expression relative to $\beta$-actin is shown in the histogram. ${ }^{* * *} \mathrm{P}<0.001 \mathrm{compared}$ with the EAE and $\mathrm{rAd} 5$-HK control groups. The data were statistically analyzed by one-way ANOVA with Tukey's post-hoc test and values are expressed as the mean \pm standard deviation. p.i., post-immunization; RGMa, repulsive guidance molecule a; EAE, experimental autoimmune encephalomyelitis; RNAi, RNA interference.

the experimental modelling p.i. (Fig. 3A and B). The expression of CCL5 was indicated to be in parallel with the deterioration of the clinical course of EAE (Fig. 2A).

RGMa RNAi significantly inhibits the expression of CCL5 in EAE mice. To examine whether RGMa regulates CCL5 expression in EAE mice, the expression of CCL5 mRNA and protein in EAE mice was measured by RT-qPCR and ELISA, respectively, after RGMa RNAi treatment 21 days p.i. The RGMa RNAi group exhibited a significant reduction in the basal levels of CCL5 mRNA and CCL5 serum protein expression compared with the EAE group and the rAd5-HK control group (Fig. 3C and D). These results suggested that CCL5 is an important downstream cytokine effector of RGMa in EAE mice.

RGMa regulates the expression of CCL5 $\mathrm{mRNA}$ in endothelial cells. The basis of the permeability of the BBB is determined by the endothelial cell composition. A previous study demonstrated that CCL5 is involved in this process, as RGMa and CCL5 were indicated to be expressed in mouse endothelial cells (19). In the present study, it was demonstrated that RGMa led to a marked increase in the expression level of CCL5 mRNA in bEnd. 3 cells, and this effect was observed as early as $2 \mathrm{~h}$ after recombinant RGMa treatment (Fig. 4A). These findings were corroborated by the similar patterns of CCL5 expression observed in the immunofluorescence experiments (Fig. 4B).
RGMa regulates the expression of CCL5 through a $B M P$-receptor-dependent pathway. It has not been previously established whether RGMa-BMP signalling inhibits CCL5 expression. Incubating the cells with noggin, an extracellular antagonist of BMP ligand, led to a marked inhibition of CCL5 mRNA expression (Fig. 4C). These results confirmed that CCL5 is regulated by RGMa or BMP in endothelial cells. Furthermore, the addition of exogenous noggin abolished the induction effect of RGMa on CCL5 mRNA (Fig. 4D). Taken together, these results suggested that RGMa enhances the expression of CCL5 through BMP receptor signalling.

\section{Discussion}

The present study demonstrated a crucial role for RGMa in the development of EAE. First, inhibition of RGMa by RNAi led to a marked improvement in neurological functions of EAE mice. Furthermore, RGMa was indicated to regulate the expression of CCL5 both in EAE mice and in endothelial cells.

A pathological hallmark of MS is infiltration of immune cells across the BBB into the CNS, which subsequently causes myelin destruction and axonal injury (20). Muramatsu et al (3) demonstrated that neutralizing antibodies against RGMa attenuated the clinical symptoms of mouse MOG-induced EAE, including a reduced invasion rate of inflammatory cells into the $\mathrm{CNS}$, although the exact mechanism underlying the association between RGMa and inflammation remained elusive. In the 


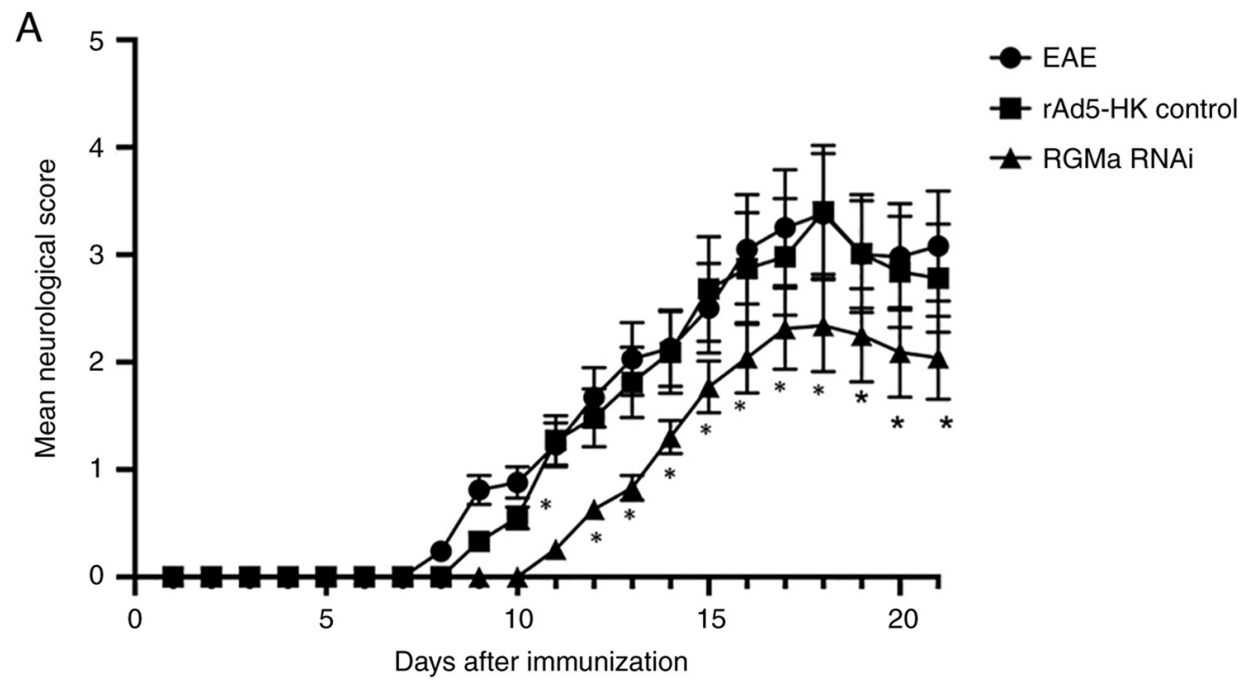

$\mathrm{B}$

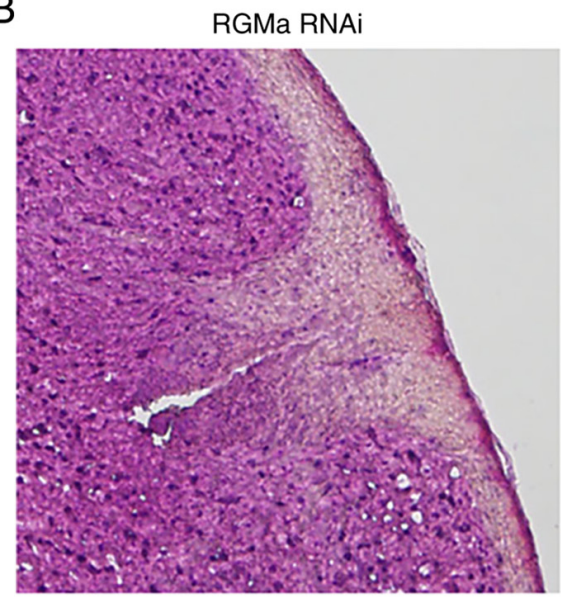

C
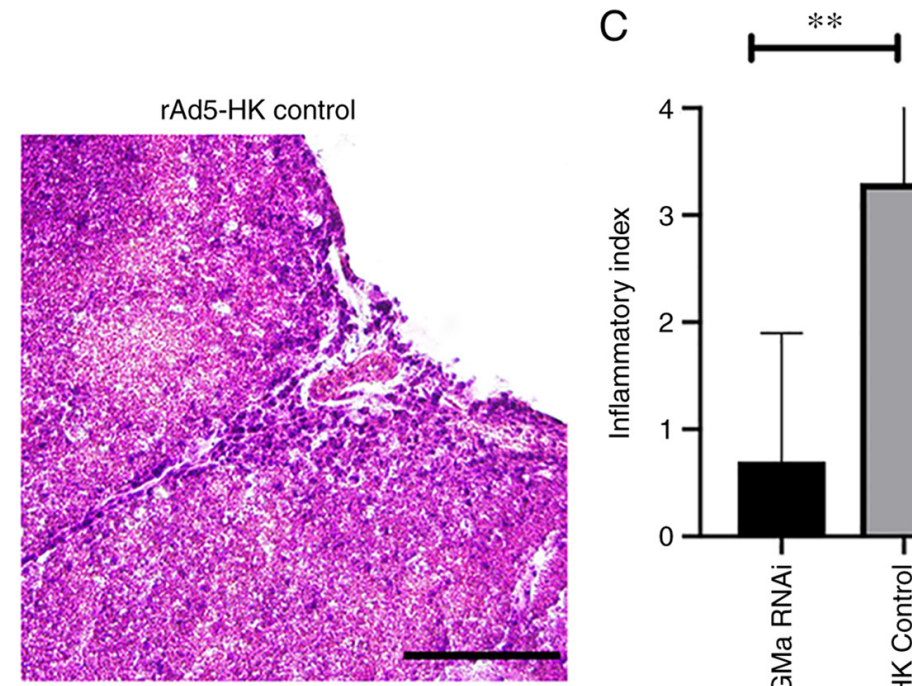

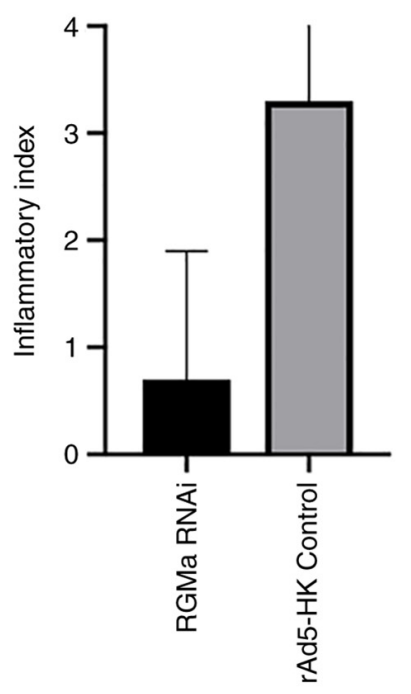

Figure 2. RGMa RNAi inhibits the deterioration of neurological function in EAE mice. The neurological function of EAE mice was evaluated according to the mean neurological scores from the first day p.i. to 21 days p.i. (A) Mice in the RGMa RNAi group had a more delayed onset of EAE (after day 11 p.i.) compared with both the EAE group (day 8 p.i.) and the rAd5-HK control group (day 9 p.i.). RGMa RNAi also significantly reduced the clinical severity of the disease on EAE after day 11 p.i., as assessed by the mean neurological scores. (B) Haematoxylin and eosin staining of the anterior columns of the cervical spinal cord in the RGMa RNAi and the rAd5-HK control groups (scale bar, $100 \mu \mathrm{m}$ ). (C) Histological scores for the inflammatory lesions. Values are expressed as the mean \pm standard deviation. $\mathrm{P}<0.05$ compared with the rAd5-HK control group (these data were analyzed by one way ANOVA with Tukey's post-hoc test); ${ }^{* * *} \mathrm{P}<0.01$ compared with the rAd5-HK control group (these data were analyzed by Student's t-test). p.i., post-immunization; RGMa, repulsive guidance molecule a; EAE, experimental autoimmune encephalomyelitis; RNAi, RNA interference.

present study, it was indicated that specific suppression of RGMa by RNAi led to a marked inhibition of the expression of RGMa and this was associated with both a significant delay in the onset of EAE and an alleviated disease course, findings which are consistent with those of the previous study. Subsequently, the present study further focused on exploring the putative role of CCL5 in the underlying mechanism.

CCL5 induces the migration of T cells across the BBB (21) and fulfils an important role in the adhesion of leukocytes in the brain microcirculation in EAE (22), which are crucial steps in the pathogenesis of MS (23). The endogenous level of CCL5 is almost undetectable in the cerebrospinal fluid of healthy individuals; however, the level of CCL5 increases markedly both at the onset and during the progression of MS (24) and during the disease progression of EAE mice (25). Knockout of CCR5 led to a marked improvement in the clinical scoring and neurological function of EAE mice, and suppressed the expression of inflammatory mediators, including IL- $1 \beta$, TNF- $\alpha$, IFN- $\gamma$ and MCP-1 (11). In the present study, the results revealed that RGMa regulates the expression of both the mRNA levels in the spinal cord and serum protein levels of CCL5 in EAE mice, suggesting that the alleviation of the disease severity in EAE via inhibition of RGMa is at least partially dependent on the regulation of CCL5. In terms of the planning of the in vitro experiments in the present study, endothelial cells were chosen, since these form an essential component in BBB permeability and migration of activated leukocytes. It was indicated that CCL5 expression is upregulated by RGMa in endothelial cells, which suggested that RGMa may improve T-cell activation and inflammation via regulating the expression of CCL5. However, further studies 

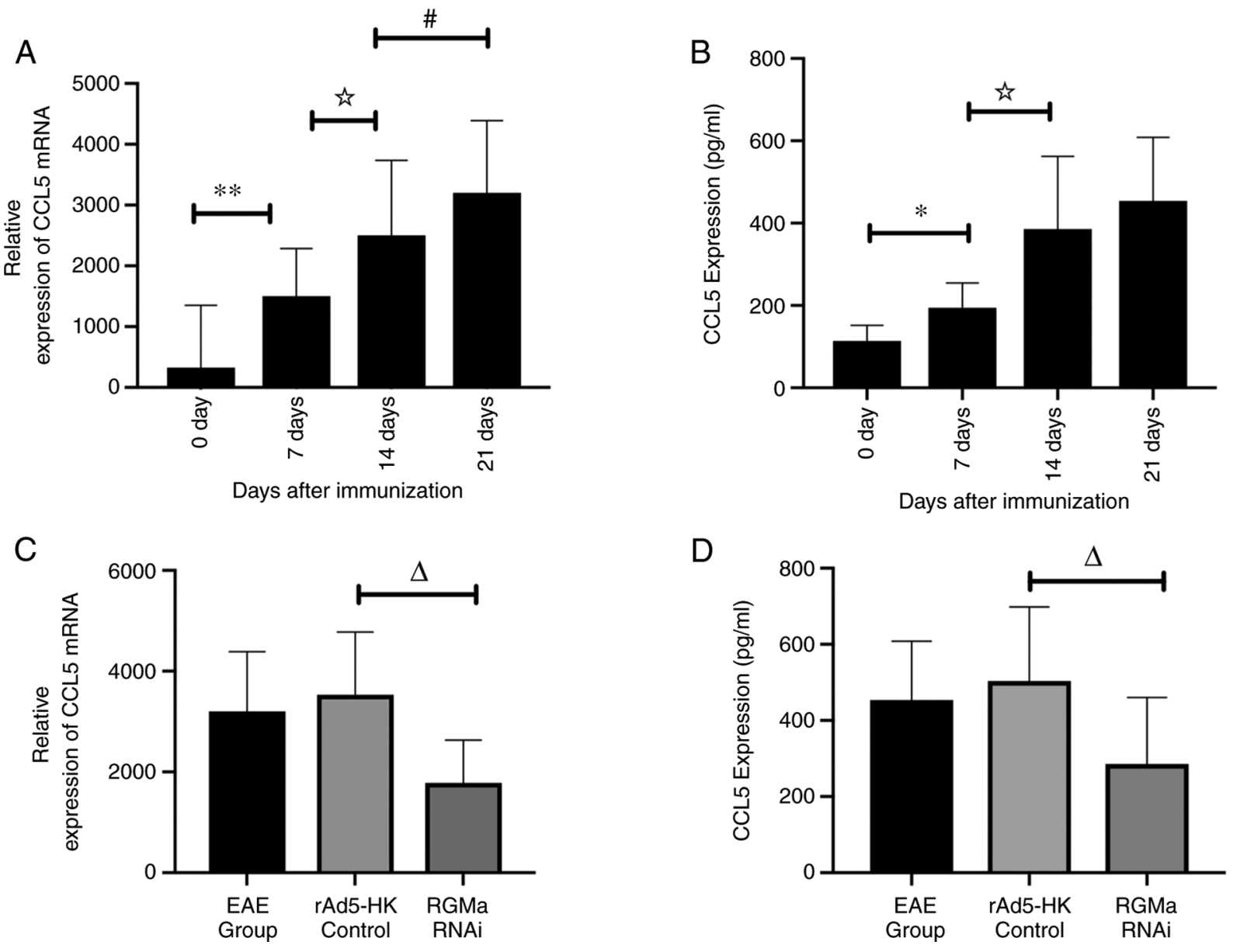

Figure 3. Expression of CCL5 in different stages of disease progression in EAE mice, as assessed by RT-qPCR and ELISA, and the inhibitory effects of RGMa RNAi on the expression of CCL5 in EAE mice. (A) CCL5 mRNA levels in the spinal cord were upregulated p.i., as determined by RT-qPCR analysis. (B) CCL5 protein levels in the serum of EAE mice showed a similar trend in increasing with the expression of CCL5 mRNA as EAE progressed, as determined by ELISA. (C) CCL5 mRNA in the spinal cord was evaluated by RT-qPCR in EAE mice at 21 days p.i. CCL5 mRNA levels in the spinal cord of the RGMa RNAi group were significantly decreased compared with the rAd5-HK control group. (D) CCL5 protein levels in the serum of EAE mice were subjected to ELISA at 21 days p.i. CCL5 protein expression levels in the RGMa RNAi group were significantly decreased compared with the rAd5-HK control group. ${ }^{*}<<0.05$ and ${ }^{* *} \mathrm{P}<0.01 ;{ }^{*} \mathrm{P}<0.05 ;{ }^{*} \mathrm{P}<0.05 ;{ }^{\circ} \mathrm{P}<0.01$. The data were analyzed by one-way ANOVA with Tukey's post-hoc test and are expressed as the mean \pm standard deviation. p.i., post-immunization; RGMa, repulsive guidance molecule a; EAE, experimental autoimmune encephalomyelitis; CCL5, C-C motif chemokine ligand 5; RNAi, RNA interference; RT-qPCR, reverse transcription-quantitative PCR.

are required to explore whether RGMa may directly impair BBB function via CCL5 regulation (such as investigating the role of claudin-5 or performing a BBB permeability assay) and examining the effects on inflammatory mediators downstream would also be necessary to further validate the results. On the other hand, several other chemokines and cytokines factors besides CCL5 that are also involved in the pathogenesis of MS and are expected to be modulated by RGMa. Further studies by our group will focus on the regulatory interactions of RGMa and those cytokines in oligodendrocytes or lymphocytes in vitro.

Neogenin has been recognized as a classical receptor of RGMa $(26,27)$. Furthermore, RGMa is also a BMP co-receptor, although unlike the well-known neogenin pathway, the biological role of the RGMa-BMP receptor pathway has not been well investigated to date. Using in vitro endothelial cell experiments, it was suggested that RMGa activates CCL5 in a BMP-receptor signalling-dependent manner. Noggin, a specific BMP receptor inhibitor, abolished the role mediated by RGMa in increasing the expression of CCL5 both at the mRNA and protein levels. Although the exact mechanism of the RGMa-BMP receptor pathway requires further research for its complete characterization, including the identification of the specific subtypes of BMP receptors that are involved, the present study was, to the best of our knowledge, the first attempt to clarify the downstream inflammatory molecules in the RGMa-BMP pathway.

In the present study, it was demonstrated that RGMa leads to an increase in CCL5 expression in a BMP ligand-dependent manner both in vivo (in EAE mice) and in vitro (in endothelial cells). This study thereby defined a previously unknown role of RGMa in modulating a chemokine, i.e. CCL5, via the BMP signalling pathway. In consideration of the role of CCL5 in lymphocyte migration, the results of the present study also potentially underline the mechanism of BBB permeability mediated by RGMa. The novel role of RGMa that was uncovered has revealed that RMGa may be a promising therapeutic target for the treatment of MS.

\section{Acknowledgements}

Not applicable. 

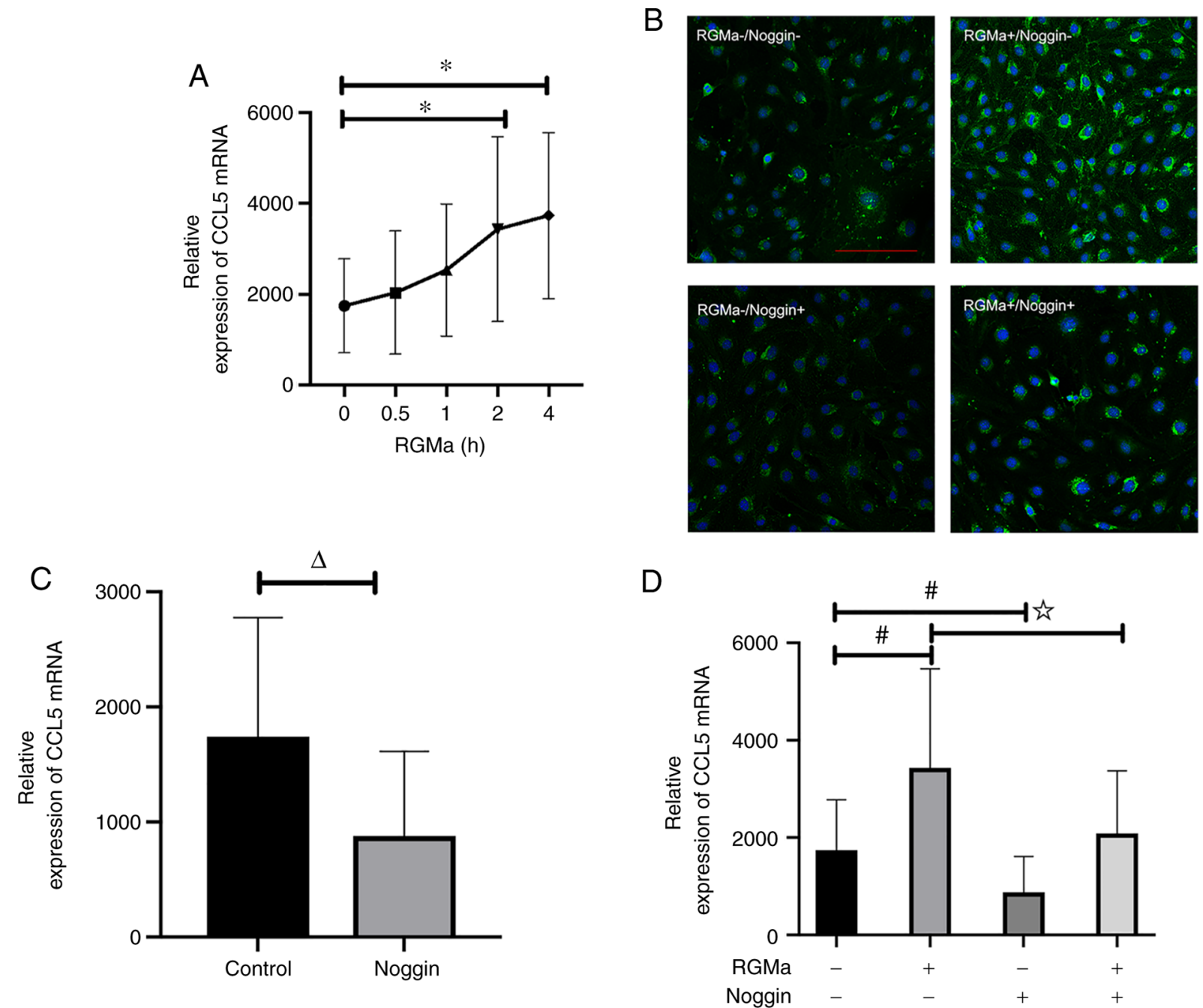

Figure 4. CCL5 mRNA is regulated by RGMa in endothelial cells. (A) CCL5 mRNA expression was upregulated by RGMa. (B) CCL5 expression was detected by immunofluorescence assay (scale bar, $100 \mu \mathrm{m}$ ). (C) CCL5 mRNA expression was inhibited by noggin, a specific BMP ligand antagonist. (D) Inactivation of BMP ligands by noggin negated the increase in CCL5 mRNA expression that was induced upon RGMa addition. Values are expressed as the mean \pm standard deviation. ${ }^{*} \mathrm{P}<0.05$ compared with the 0 h group; ${ }^{\wedge} \mathrm{P}<0.05 ;{ }^{*} \mathrm{P}<0.05$; ${ }^{\mathrm{A}} \mathrm{P}<0.05$. RGMa, repulsive guidance molecule a; CCL5, C-C motif chemokine ligand 5; BMP, bone morphogenetic protein.

\section{Funding}

The present study was supported by the Science and Technology Project of Chongqing Education Committee (grant no. KJ1600210), the National Natural Science Foundation of China (grant no. 81701191) and the National Key Clinical Specialties Construction Program of China.

\section{Availability of data and materials}

The datasets used and/or analyzed during the current study are available from the corresponding author on reasonable request.

\section{Authors' contributions}

All authors performed the experiments. ST drafted the manuscript. BS analyzed and interpreted the data. TT performed RNAi and cell culture. WY performed EAE, qPCR and immunofluorescence. RZ performed western blot analysis. XQ and JF designed the study and revised the manuscript. All authors read and approved the final manuscript. ST and XQ checked and confirmed the authenticity of the raw data.

\section{Ethics approval and consent to participate}

All animal procedures were approved by the Ethics Committee of the First Affiliated Hospital of Chongqing Medical University (Chongqing, China) and all procedures were performed in accordance with the Guide for the Care and Use of Laboratory Animals (12).

\section{Patient consent for publication}

Not applicable.

\section{Competing interests}

The authors declare that they have no competing interests. 


\section{References}

1. Siebold C, Yamashita T, Monnier PP, Mueller BK and Pasterkamp RJ: RGMs: Structural insights, molecular regulation, and downstream signaling. Trends Cell Biol 27: 365-378, 2017.

2. Monnier PP, Sierra A, Macchi P, Deitinghoff L, Andersen JS, Mann M, Flad M, Hornberger MR, Stahl B, Bonhoeffer F and Mueller BK: RGM is a repulsive guidance molecule for retinal axons. Nature 419: 392-395, 2002.

3. Muramatsu R, Kubo T, Mori M, Nakamura Y, Fujita Y, Akutsu T, Okuno T, Taniguchi J, Kumanogoh A, Yoshida M, et al: RGMa modulates $\mathrm{T}$ cell responses and is involved in autoimmune encephalomyelitis. Nat Med 17: 488-494, 2011.

4. Fujita Y and Yamashita T: The roles of RGMa-neogenin signaling in inflammation and angiogenesis. Inflamm Regen 37: 6, 2017.

5. Harada K, Fujita Y and Yamashita T: Repulsive guidance molecule A suppresses angiogenesis. Biochem Biophys Res Commun 469: 993-999, 2016.

6. Minagar A and Alexander JS: Blood-brain barrier disruption in multiple sclerosis. Mult Scler 9: 540-549, 2003.

7. Wang Y, Zhang R, Xing X, Guo J, Xie F, Zhang G and Qin X: Repulsive guidance molecule a suppresses angiogenesis after ischemia/reperfusion injury of middle cerebral artery occlusion in rats. Neurosci Lett 662: 318-323, 2018

8. Cheng W and Chen G: Chemokines and chemokine receptors in multiple sclerosis. Mediators Inflamm 2014: 659206, 2014.

9. Pittaluga A: CCL5-glutamate cross-talk in astrocyte-neuron communication in multiple sclerosis. Front Immunol 8: 1079, 2017

10. Tang S, Xiang T, Huang S, Zhou J, Wang Z, Xie R, Long H and Zhu B: Ovarian cancer stem-like cells differentiate into endothelial cells and participate in tumor angiogenesis through autocrine CCL5 signaling. Cancer Lett 376: 137-147, 2016.

11. Gu SM, Park MH, Yun HM, Han SB, Oh KW, Son DJ, Yun JS and Hong JT: CCR5 knockout suppresses experimental autoimmune encephalomyelitis in C57BL/6 mice. Oncotarget 7: 15382-15393, 2016.

12. The National Academies Collection: Reports funded by National Institutes of Health. Guide for the Care and Use of Laboratory Animals, 8th edition. Washington (DC): National Academies Press (US); 2011.

13. Feng J, Tao T, Yan W, Chen CS and Qin X: Curcumin inhibits mitochondrial injury and apoptosis from the early stage in EAE mice. Oxid Med Cell Longev 2014: 728751, 2014.

14. Feng J, Wang T, Li Q, Wu X and Qin X: RNA interference against repulsive guidance molecule $\mathrm{A}$ improves axon sprout and neural function recovery of rats after MCAO/reperfusion. Exp Neurol 238: 235-242, 2012

15. Wang CJ, Qu CQ, Zhang J, Fu PC, Guo SG and Tang RH: Lingo-1 inhibited by RNA interference promotes functional recovery of experimental autoimmune encephalomyelitis. Anat Rec (Hoboken) 297: 2356-2363, 2014

16. Croxford JL, Feldmann M, Chernajovsky Y and Baker D Different therapeutic outcomes in experimental allergic encephalomyelitis dependent upon the mode of delivery of IL-10: A comparison of the effects of protein, adenoviral or retroviral IL-10 delivery into the central nervous system. J Immunol 166 : 4124-4130, 2001.
17. Schmittgen TD and Livak KJ: Analyzing real-time PCR data by the comparative C(T) method. Nat Protoc 3: 1101-1108, 2008.

18. Zhang G, Wang R, Cheng K, Li Q, Wang Y, Zhang R and Qin X: Repulsive guidance molecule a inhibits angiogenesis by downregulating VEGF and phosphorylated focal adhesion kinase in vitro. Front Neurol 8: 504, 2017.

19. Ouyang S, Hsuchou H, Kastin AJ, Mishra PK, Wang Y and Pan W: Leukocyte infiltration into spinal cord of EAE mice is attenuated by removal of endothelial leptin signaling. Brain Behav Immun 40: 61-73, 2014.

20. Bradl M and Hohlfeld R: Molecular pathogenesis of neuroinflammation. J Neurol Neurosurg Psychiatry 74: 1364-1370, 2003.

21. Ubogu EE, Callahan MK, Tucky BH and Ransohoff RM: CCR5 expression on monocytes and T cells: Modulation by transmigration across the blood-brain barrier in vitro. Cell Immunol 243: 19-29, 2006.

22. Dos Santos AC, Roffê E, Arantes RM, Juliano L, Pesquero JL, Pesquero JB, Bader M, Teixeira MM and Carvalho-Tavares J: Kinin B2 receptor regulates chemokines CCL2 and CCL5 expression and modulates leukocyte recruitment and pathology in experimental autoimmune encephalomyelitis (EAE) in mice. J Neuroinflammation 5: 49, 2008.

23. dos Santos AC, Barsante MM, Arantes RM, Bernard CC, Teixeira MM and Carvalho-Tavares J: CCL2 and CCL5 mediate leukocyte adhesion in experimental autoimmune encephalomyelitis-an intravital microscopy study. J Neuroimmunol 162: 122-129, 2005.

24. Sorensen TL, Tani M, Jensen J, Pierce V, Lucchinetti C, Folcik VA, Qin S, Rottman J, Sellebjerg F, Strieter RM, et al: Expression of specific chemokines and chemokine receptors in the central nervous system of multiple sclerosis patients. J Clin Invest 103: 807-815, 1999.

25. Mecha M, Feliú A, Iñigo PM, Mestre L, Carrillo-Salinas FJ and Guaza C: Cannabidiol provides long-lasting protection against the deleterious effects of inflammation in a viral model of multiple sclerosis: A role for A2A receptors. Neurobiol Dis 59: 141-150, 2013.

26. Rajagopalan S, Deitinghoff L, Davis D, Conrad S, Skutella T, Chedotal A, Mueller BK and Strittmatter SM: Neogenin mediates the action of repulsive guidance molecule. Nat Cell Biol 6: 756-762, 2004.

27. Matsunaga E, Tauszig-Delamasure S, Monnier PP, Mueller BK, Strittmatter SM, Mehlen P and Chédotal A: RGM and its receptor neogenin regulate neuronal survival. Nat Cell Biol 6: 749-755, 2004.

This work is licensed under a Creative Commons Attribution-NonCommercial-NoDerivatives 4.0 International (CC BY-NC-ND 4.0) License. 